Article

\title{
Stability of Generalized Proportional Caputo Fractional Differential Equations by Lyapunov Functions
}

\author{
Ravi Agarwal ${ }^{1} \mathbb{D}$, Snezhana Hristova ${ }^{2, * \mathbb{D}}$ and Donal $\mathrm{O}^{\prime}$ Regan $^{3}$ \\ 1 Department of Mathematics, Texas A\&M University-Kingsville, Kingsville, TX 78363, USA; \\ Ravi.Agarwal@tamuk.edu \\ 2 Faculty of Mathematics and Informatics, University of Plovdiv "Paisii Hilendarski", 4000 Plovdiv, Bulgaria \\ 3 School of Mathematical and Statistical Sciences, National University of Ireland, H91 TK33 Galway, Ireland; \\ donal.oregan@nuigalway.ie \\ * Correspondence: snehri@uni-plovdiv.bg or snehri@gmail.com
}

check for updates

Citation: Agarwal, R.; Hristova, S.; O'Regan, D. Stability of Generalized Proportional Caputo Fractional Differential Equations by Lyapunov Functions. Fractal Fract. 2022, 6, 34. https://doi.org/10.3390/fractalfract 6010034

Academic Editor: Paul Eloe

Received: 23 November 2021

Accepted: 7 January 2022

Published: 10 January 2022

Publisher's Note: MDPI stays neutral with regard to jurisdictional claims in published maps and institutional affiliations.

Copyright: (C) 2022 by the authors. Licensee MDPI, Basel, Switzerland. This article is an open access article distributed under the terms and conditions of the Creative Commons Attribution (CC BY) license (https:// creativecommons.org/licenses/by/ $4.0 /)$.

\begin{abstract}
In this paper, nonlinear nonautonomous equations with the generalized proportional Caputo fractional derivative (GPFD) are considered. Some stability properties are studied by the help of the Lyapunov functions and their GPFDs. A scalar nonlinear fractional differential equation with the GPFD is considered as a comparison equation, and some comparison results are proven. Sufficient conditions for stability and asymptotic stability were obtained. Examples illustrating the results and ideas in this paper are also provided.
\end{abstract}

Keywords: generalized proportional Caputo fractional derivative; fractional differential equations; stability; asymptotic stability; Lyapunov functions

MSC: 34A08; 34A34; 34D20

\section{Introduction}

The stability properties of solutions are an important branch in the qualitative theory of differential equations. One of the most effective and applicable methods for investigation of the stability properties of solutions is the Lyapunov approach [1]. Various types of stability for Caputo fractional differential equations by the Caputo fractional derivative of Lyapunov functions have been presented and discussed (see, for example, [2-7]). Recently, in 2017 [8], the generalized proportional Caputo fractional derivative (GPFD) was introduced and applied to various problems. This derivative is a generalization of the Caputo fractional derivative. Note that the GPFE is similar to the so-called tempered fractional derivative. For some results concerning the GPFR and the differential equations with the GPFD, as well as its applications, we refer the reader to [9-13]. However, the study of the stability properties of the solutions of fractional differential equations with the GPFD is at its initial stage (see, for example, [14]). Note that, in contrast to ordinary derivatives, fractional derivatives depend significantly on the initial time point $t_{0}$, which is equal to the lower limit of the derivative ([15-20]). Therefore, any change of the initial time leads to a change of the fractional derivative and the corresponding fractional differential differential equation. For this reason, we study only stability and asymptotic stability instead of the uniform ones.

In this paper, we used appropriate Lyapunov functions and their GPFD among the solutions of the appropriate equation. Comparison results with the scalar fractional differential equations with the GPFD and Lyapunov functions were obtained. Several sufficient conditions for stability and asymptotic stability are presented. Some examples illustrate the ideas and results in this paper. 


\section{Notes on Fractional Calculus}

Let $u:[a, T] \rightarrow \mathbb{R}$ (if $T=\infty$, then the interval is half-open). The generalized proportional fractional integral is defined by (as long as all integrals are well defined; see [10]):

$$
\left({ }_{a} I^{\alpha, \rho} u\right)(t)=\frac{1}{\rho^{\alpha} \Gamma(\alpha)} \int_{a}^{t} e^{\frac{\rho-1}{\rho}(t-s)}(t-s)^{\alpha-1} u(s) d s, \quad t \in(a, T], \alpha \geq 0, \rho \in(0,1],
$$

and the generalized Caputo proportional fractional derivative (GPFD) is defined by (as long as all integrals are well defined; see [10]):

$$
\begin{aligned}
& \left({ }_{a}^{C} D^{\alpha, \rho} u\right)(t)=\left({ }_{a} I^{1-\alpha, \rho}\left(D^{1, \rho} u\right)\right)(t) \\
& =\frac{1}{\rho^{1-\alpha} \Gamma(1-\alpha)} \int_{a}^{t} e^{\frac{\rho-1}{\rho}(t-s)}(t-s)^{-\alpha}\left(D^{1, \rho} u\right)(s) d s, \\
& \text { for } t \in(a, T], \quad \alpha \in(0,1), \rho \in(0,1],
\end{aligned}
$$

where $\left(D^{1, \rho} u\right)(t)=\left(D^{\rho} u\right)(t)=(1-\rho) u(t)+\rho u^{\prime}(t)$

Remark 1. Note that in the case $\rho=1$, the GPFE is reduced to the Caputo fractional derivative: $\left({ }_{a}^{C} D^{\alpha, 1} u\right)(t)={ }_{a}^{C} D^{\alpha} u(t)$.

Remark 2. The GPFE given by (2) could be generalized for any function $u \in C\left([a, b], \mathbb{R}^{n}\right)$ via a componentwise approach.

Lemma 1 (Theorem $5.3[10])$. For $\rho \in(0,1]$ and $\alpha \in(0,1)$, we have:

$$
\left({ }_{a} I^{\alpha, \rho}\left({ }_{a}^{C} D^{\alpha, \rho} u\right)\right)(t)=u(t)-u(a) e^{\frac{\rho-1}{\rho}(t-a)}
$$

Lemma 2 (Theorem $5.2[10])$. For $\rho \in(0,1]$ and $\alpha \in(0,1)$, we have:

$$
\left.\left({ }_{a} I^{\alpha, \rho} e^{\frac{\rho-1}{\rho} t}(t-a)^{\beta-1}\right)(\tau)=\frac{\Gamma(\beta)}{\rho^{\alpha} \Gamma(\beta+\alpha)}\right) e^{\frac{\rho-1}{\rho} \tau}(\tau-a)^{\beta-1+\alpha} \quad \beta>0 .
$$

Remark 3. If $\rho \in(0,1)$, then $\left({ }_{a}^{C} D^{\alpha, \rho} c\right)(t) \not \equiv 0$, where $c$ is a nonzero constant.

Remark 4. The relation:

$$
\left({ }_{a}^{C} D^{\alpha, \rho} e^{\frac{\rho-1}{\rho}(\cdot)}\right)(t)=0 \text { for } t>a
$$

is known from [10], Remark 3.2.

We used the result given in Example 5.7 of [10] (with necessary slight corrections).

Consider the initial-value problem for the scalar linear fractional differential equation with the GPFD:

$$
\left({ }_{a}^{C} D^{\alpha, \rho} y\right)(t)=\lambda y(t), \quad y(a)=y_{0}, t \in[a, T], \alpha \in(0,1), \rho \in(0,1]
$$

with $y_{0} \in \mathbb{R}$.

Lemma 3. The initial-value problem (6) has a solution:

$$
y(t)=y_{0} e^{\frac{\rho-1}{\rho}(t-a)} E_{\alpha}\left(\lambda\left(\frac{t-a}{\rho}\right)^{\alpha}\right),
$$

where $E_{\alpha}(t)$ is the Mittag-Leffler function. 
Lemma 4 ([14]). Let the function $u \in C^{1}([a, T], \mathbb{R}), T \leq \infty$ and $\alpha \in(0,1), \rho \in(0,1]$ be two reals. Then,

$$
\left({ }_{a}^{C} D^{\alpha, \rho} u^{2}\right)(t) \leq 2 u(t)\left({ }_{a}^{C} D^{\alpha, \rho} u\right)(t), \quad t \in(a, T] .
$$

\section{Statement of the Problem}

Consider the following nonlinear system of fractional differential equations with the GPFE:

$$
\left({ }_{t_{0}}^{C} D^{\alpha, \rho} x\right)(t)=f(t, x(t)), \quad \text { for } t>t_{0}, \quad \alpha \in(0,1), \rho \in(0,1]
$$

with the initial condition:

$$
x\left(t_{0}\right)=x_{0},
$$

where $t_{0}>0, x_{0} \in \mathbb{R}^{n}, f \in C\left(\left[t_{0}, \infty\right) \times \mathbb{R}^{n}, \mathbb{R}^{n}\right)$.

In our paper, we assumed that $f(t, 0) \equiv 0$, so the zero is a solution of (9).

We denote the solution of (9), (10) by $x\left(t ; t_{0}, x_{0}\right)$. We assumed in the paper the initialvalue problem (9), (10) has a solution defined for $t \geq t_{0}$ for any initial value $x_{0} \in \mathbb{R}^{n}$. Some existence results were given in [21,22].

Definition 1. The zero solution of (9) is:

- Stable if for every $\epsilon>0$, there exist $\delta=\delta(\epsilon)>0$ such that for any $x_{0} \in \mathbb{R}^{n}$, the inequality $\left\|x_{0}\right\|<\delta$ implies $\left\|x\left(t ; t_{0}, x_{0}\right)\right\|<\epsilon$ for $t \geq t_{0}$;

- Attractive if there exists $B>0$ such that for any $x_{0} \in \mathbb{R}^{n}$ with $\left\|x_{0}\right\|<B$ and for every $\epsilon>0$, there exists $\tilde{T}=\tilde{T}(\epsilon)>0$ such that $\left\|x\left(t ; t_{0}, x_{0}\right)\right\|<\epsilon$ for $t \geq t_{0}+\tilde{T}$;

- Asymptotically stable if the zero solution is stable and attractive.

Define the following set:

$$
\mathcal{K}=\{a \in C([0, \infty),[0, \infty)): a \text { is strictly increasing and } a(0)=0\} .
$$

Consider the comparison scalar fractional differential equation with the GPFE:

$$
\left({ }_{t_{0}}^{C} D^{\alpha, \rho} y\right)(t)=h(t, y(t)), \quad t>t_{0},
$$

where $h:\left[t_{0}, \infty\right) \times \mathbb{R} \rightarrow \mathbb{R}, h(t, 0) \equiv 0$. We assumed there exists a small enough number $L>0$ such that the equation $\left({ }_{t_{0}}^{C} D^{\alpha, \rho} y\right)(t)=h(t, y(t))+\eta e^{\frac{\rho-1}{\rho}(t-s)}, \eta \in(0, L]$ with $y\left(t_{0}\right)=y_{0}$ has a solution $y\left(t ; t_{0}, y_{0}, \eta\right)$ where $y_{0} \in \mathbb{R}$.

Example 1. Let us consider the scalar fractional differential equation:

$$
\left({ }_{t_{0}}^{C} D^{\alpha, \rho} y\right)(t)=-b u, \quad y\left(t_{0}\right)=y_{0},
$$

where $y_{0} \in \mathbb{R}, b>0$.

According to Lemma 3, the solution of (12) is:

$$
y(t)=y_{0} e^{\frac{\rho-1}{\rho}\left(t-t_{0}\right)} E_{\alpha}\left(-b\left(\frac{t-t_{0}}{\rho}\right)^{\alpha}\right) .
$$

From (13) and the inequality $0<E_{\alpha}\left(-b\left(t-t_{0}\right)^{q}\right)<1, t \geq t_{0}$, we obtain:

$$
|y(t)| \leq\left|y_{0}\right| .
$$

Inequality (14) proves that the zero solution of (12) is stable.

We define a class of Lyapunov functions. 
Definition 2. Let $V(t, x) \in C\left(\left[t_{0}, T\right) \times \Delta, \mathbb{R}_{+}\right)$be locally Lipschitzian with respect to its second argument and $V(t, 0) \equiv 0$, where $t_{0}, T \in \mathbb{R}_{+}: T>t_{0}, \Delta \subset \mathbb{R}^{n}, 0 \in \Delta$. Then, we say $V \in \Lambda\left(\left[t_{0}, T\right), \Delta\right)$.

We used the generalized proportional Caputo fractional derivative of the Lyapunov function for any solution of the system of fractional equations. Note that in the case of Caputo fractional derivatives, some authors $[3,6,7]$ have used a similar approach.

\section{Comparison Results}

Lemma 5. Let $u \in C\left(\left[t_{0}, T\right], \mathbb{R}\right)$, and there exists a point $t^{*} \in\left(t_{0}, T\right]$, such that $u\left(t^{*}\right)=0$ and $u(t)<0$ for $t_{0} \leq t<t^{*}$. Then, if the generalized proportional Caputo fractional derivative of $u$ exists at $t^{*}$, then the inequality $\left.\left({ }_{t_{0}}^{C} D^{\alpha, \rho} u\right)(t)\right|_{t=t^{*}}>0$ holds.

Proof. From the definition of the GPFD and integration by parts, we obtain:

$$
\begin{aligned}
&\left.\left({ }_{t_{0}}^{C} D^{\alpha, \rho} u\right)(t)\right|_{t=t^{*}}=\frac{1}{\rho^{1-\alpha} \Gamma(1-\alpha)} \int_{t_{0}}^{t^{*}} e^{\frac{\rho-1}{\rho}\left(t^{*}-s\right)} \frac{(1-\rho) u(s)+\rho u^{\prime}(s)}{\left(t^{*}-s\right)^{\alpha}} d s \\
&=\frac{\rho^{\alpha}}{\Gamma(1-\alpha)} \int_{t_{0}}^{t^{*}}\left\{\frac{1-\rho}{\rho} e^{\frac{\rho-1}{\rho}\left(t^{*}-s\right)} \frac{u(s)}{\left(t^{*}-s\right)^{\alpha}}+e^{\frac{\rho-1}{\rho}(t-s)} \frac{u^{\prime}(s)}{\left(t^{*}-s\right)^{\alpha}}\right\} d s \\
&=\frac{\rho^{\alpha}}{\Gamma(1-\alpha)}\left\{\int_{t_{0}}^{t^{*}} \frac{d}{d s}\left(e^{\frac{\rho-1}{\rho}\left(t^{*}-s\right)}\right) \frac{u(s)}{\left(t^{*}-s\right)^{\alpha}} d s\right. \\
&\left.\quad+e^{\frac{\rho-1}{\rho}\left(t^{*}-s\right)}\left[\frac{d}{d s}\left(\frac{u(s)}{\left(t^{*}-s\right)^{\alpha}}\right)-\frac{\alpha u(s)}{\left(t^{*}-s\right)^{\alpha+1}}\right] d s\right\} \\
&=\frac{\rho^{\alpha}}{\Gamma(1-\alpha)} \int_{t_{0}}^{t^{*}} \frac{d}{d s}\left(e^{\frac{\rho-1}{\rho}\left(t^{*}-s\right)} \frac{u(s)}{\left(t^{*}-s\right)^{\alpha}}\right) d s \\
& \quad-\frac{\alpha \rho^{\alpha}}{\Gamma(1-\alpha)} \int_{t_{0}}^{t^{*}} e^{\frac{\rho-1}{\rho}\left(t^{*}-s\right)} \frac{u(s)}{\left(t^{*}-s\right)^{\alpha+1}} d s \\
&=\frac{\rho^{\alpha}}{\Gamma(1-\alpha)} \lim _{s \rightarrow t^{*-}} e^{\frac{\rho-1}{\rho}\left(t^{*}-s\right)} \frac{u(s)}{\left(t^{*}-s\right)^{\alpha}}-e^{\frac{\rho-1}{\rho}\left(t^{*}-t_{0}\right)} \frac{u\left(t_{0}\right)}{\left(t^{*}-t_{0}\right)^{\alpha}} \\
&+\frac{\rho^{\alpha}}{\Gamma(-\alpha)} \int_{t_{0}}^{t^{*}} e^{\frac{\rho-1}{\rho}\left(t^{*}-s\right)} \frac{u(s)}{\left(t^{*}-s\right)^{1+\alpha}} d s .
\end{aligned}
$$

Using $\Gamma(-\alpha)<0$ for $\alpha \in(0,1), u(t)<0$ for $t_{0} \leq t<t^{*}$, and L'Hôpital's rule applied to:

$$
\left.\lim _{s \rightarrow t^{*-}} \frac{e^{\frac{\rho-1}{\rho}\left(t^{*}-s\right)} u(s)}{\left(t^{*}-s\right)^{\alpha}}=\lim _{s \rightarrow t^{*-}}\left[e^{\frac{\rho-1}{\rho}\left(t^{*}-s\right)}\right)^{\prime} u(s)+u^{\prime}(s) e^{\frac{\rho-1}{\rho}\left(t^{*}-s\right)}\right] \alpha\left(t^{*}-s\right)^{1-\alpha}=0,
$$

we obtain:

$$
\left.\left({ }_{t_{0}}^{C} D^{\alpha, \rho} u\right)(t)\right|_{t=t^{*}}=-e^{\frac{\rho-1}{\rho}\left(t^{*}-t_{0}\right)} \frac{u\left(t_{0}\right)}{\left(t^{*}-t_{0}\right)^{\alpha}}+\frac{\rho^{\alpha}}{\Gamma(-\alpha)} \int_{t_{0}}^{t^{*}} e^{\frac{\rho-1}{\rho}\left(t^{*}-s\right)} \frac{u(s)}{\left(t^{*}-s\right)^{\alpha+1}} d s>0 .
$$

We now obtain a comparison result.

Lemma 6. (Comparison result). Assume:

1. The function $\tilde{x}()=.x\left(. ; t_{0}, x_{0}\right) \in \Delta$ is a solution of $(9)$ defined on $\left[t_{0}, T\right]$, where $\Delta \subset \mathbb{R}^{n}, 0 \in \Delta$, and $T: t_{0}<T \leq \infty$ is a given constant, $x_{0} \in \Delta$;

2. The function $h \in C\left(\left[t_{0}, T\right] \times \mathbb{R}, \mathbb{R}\right)$; 
3. The function $V \in \Lambda\left(\left[t_{0}, T\right], \Delta\right)$; the generalized proportional Caputo fractional derivative exists for any point $t \in\left(t_{0}, T\right)$, and the inequality:

$$
\left({ }_{t_{0}}^{C} D^{\alpha, \rho} V(., \tilde{x}(.))\right)(t) \leq h(t, V(t, \tilde{x}(t))), t \in\left(t_{0}, T\right]
$$

holds;

4. The function $y^{*}(t)=y\left(t ; t_{0}, y_{0}\right)$ is the maximal solution of $(11)$ on $\left[t_{0}, T\right]$.

Then, the inequality $V\left(t_{0}, x_{0}\right) \leq y_{0}$ implies $V(t, \tilde{x}(t)) \leq y^{*}(t)$ for $t \in\left[t_{0}, T\right]$.

Proof. Consider:

$$
\left({ }_{t_{0}}^{C} D^{\alpha, \rho} y\right)(t)=h(t, y(t))+\eta e^{\frac{\rho-1}{\rho}\left(t-t_{0}\right)}, \quad t \in\left[t_{0}, T\right], \quad y\left(t_{0}\right)=y_{0}+\eta,
$$

where $\eta \leq L$, as described after (11).

Take the generalized proportional integral operator on both sides of Equation (17), apply Lemma 2 with $\beta=1$ and Lemma 1 , and obtain for the solution: $y(t, \eta)$ of (17):

$$
\begin{aligned}
y(t, \eta)= & y\left(t_{0}\right) e^{\frac{\rho-1}{\rho}\left(t-t_{0}\right)}+\left(t_{0} I^{\alpha, \rho}(h(., y(., \eta))+\eta)\right)(t) \\
= & \left(y_{0}+\eta\right) e^{\frac{\rho-1}{\rho}\left(t-t_{0}\right)}+\left(t_{0} I^{\alpha, \rho}(h(., y(., \eta)))(t)+\frac{\eta}{\rho^{\alpha} \Gamma(1+\alpha)}\right) e^{\frac{\rho-1}{\rho}\left(t-t_{0}\right)}\left(t-t_{0}\right)^{\alpha} \\
= & \left.\left(y_{0}+\eta\right) e^{\frac{\rho-1}{\rho}\left(t-t_{0}\right)}+\frac{\eta}{\rho^{\alpha} \Gamma(1+\alpha)}\right) e^{\frac{\rho-1}{\rho}\left(t-t_{0}\right)}\left(t-t_{0}\right)^{\alpha} \\
& +\frac{1}{\rho^{\alpha} \Gamma(\alpha)} \int_{t_{0}}^{t} e^{\frac{\rho-1}{\rho}(t-s)}(t-s)^{\alpha-1} h(s, y(s, \eta)) d s \quad \text { for } t \in\left[t_{0}, T\right] .
\end{aligned}
$$

Consider the function $\mu(t) \in C\left(\left[t_{0}, T\right], \mathbb{R}_{+}\right)$defined by $\mu(t)=V(t, \tilde{x}(t))$. We prove that:

$$
\mu(t)<y(t, \eta) \text { for } t \in\left[t_{0}, T\right] .
$$

The inequality (19) holds for $t=t_{0}$ because $\mu\left(t_{0}\right)=V\left(t_{0}, x_{0}\right) \leq y_{0}<y_{0}+\eta=y\left(t_{0}, \eta\right)$. Assume that Inequality (19) is not true. Then, there exists a point $\tau$ such that $\mu(\tau)=y(\tau, \eta)$, $\mu(t)<y(t, \eta)$ for $t \in\left[t_{0}, \tau\right)$. Now, Lemma 6 (applied to $\left.\mu(t)-y(t, \eta)\right)$ yields $\left({ }_{t_{0}}^{C} D^{\alpha, \rho} \mu()-\right.$. $y(., \eta))\left.(t)\right|_{t=\tau}>0$, i.e.,

$$
\left.\left({ }_{t_{0}}^{C} D^{\alpha, \rho} \mu\right)(t)\right|_{t=\tau}>\left.\left({ }_{t_{0}}^{C} D^{\alpha, \rho} y(., \eta)\right)(t)\right|_{t=\tau}=h(\tau, y(\tau, \eta))+\eta>h(\tau, \mu(\tau)) .
$$

From Condition 3 with $t=\tau$, the inequality $\left.\left({ }_{t_{0}}^{C} D^{\alpha, \rho} V\right)(., \tilde{x}()).\right)(t)=\left.\left({ }_{t_{0}}^{C} D^{\alpha, \rho} \mu\right)(t)\right|_{t=\tau} \leq$ $h(\tau, V(\tau, \tilde{x}(\tau)))=h(\tau, \mu((\tau))$ holds. The obtained contradiction proves (19).

We now show that if $\eta_{2}<\eta_{1}$, then:

$$
y\left(t, \eta_{2}\right)<y\left(t, \eta_{1}\right) \text { for } t \in\left[t_{0}, T\right] .
$$

Inequality (21) holds for $t=t_{0}$. Assume that Inequality (21) is not true. Then, there exists a point $t^{*}: y\left(t^{*}, \eta_{2}\right)=y\left(t^{*}, \eta_{1}\right)$ and $y\left(t, \eta_{2}\right)<y\left(t, \eta_{1}\right)$ for $t \in\left[t_{0}, t^{*}\right)$. From Lemma 6 (applied to $\left.y\left(t, \eta_{2}\right)-y\left(t, \eta_{1}\right)\right)$, we obtain $\left(\left.{ }_{t_{0}}^{C} D^{\alpha, \rho}\left(y\left(., \eta_{2}\right)-y\left(., \eta_{1}\right)\right)(t)\right|_{t=t^{*}}>0\right.$. However:

$$
\begin{aligned}
& \left(\left.{ }_{t_{0}}^{C} D^{\alpha, \rho}\left(y\left(., \eta_{2}\right)-y\left(., \eta_{1}\right)\right)(t)\right|_{t=t^{*}}\right. \\
& =\left(\left.{ }_{t_{0}}^{C} D^{\alpha, \rho}\left(y\left(., \eta_{2}\right)\right)(t)\right|_{t=t^{*}}-\left(\left.{ }_{t_{0}}^{C} D^{\alpha, \rho}\left(y\left(., \eta_{1}\right)\right)(t)\right|_{t=t^{*}}\right.\right. \\
& =h\left(t^{*}, y\left(t^{*}, \eta_{2}\right)\right)+\eta_{2} e^{\frac{\rho-1}{\rho}\left(t-t_{0}\right)}-\left[h\left(t^{*}, y\left(t^{*}, \eta_{1}\right)\right)+\eta_{1} e^{\frac{\rho-1}{\rho}\left(t-t_{0}\right)}\right] \\
& =h\left(t^{*}, y\left(t^{*}, \eta_{1}\right)\right)+\eta_{2} e^{\frac{\rho-1}{\rho}\left(t-t_{0}\right)}-\left[h\left(t^{*}, y\left(t^{*}, \eta_{1}\right)\right)+\eta_{1} e^{\frac{\rho-1}{\rho}\left(t-t_{0}\right)}\right] \\
& =\left(\eta_{2}-\eta_{1}\right) e^{\frac{\rho-1}{\rho}\left(t-t_{0}\right)}<0 .
\end{aligned}
$$

The obtained contradiction proves Inequality (21). 
From (19) and (21), it follows that the family of solutions $\{y(t, \eta)\}, t \in\left[t_{0}, T\right]$ of (17) is uniformly bounded, i.e, there exists $K>0$ with $|y(t, \eta)| \leq K$ for $(t, \eta) \in\left[t_{0}, T\right] \times[0, L]$. Denote $M=\sup \left\{|h(t, x)|:(t, x) \in\left[t_{0}, T\right] \times[-K, K]\right\}$.

Let $\left\{\eta_{j}\right\}_{j=0}^{\infty}: \eta_{j}<\eta_{j-1} \leq \eta_{0} \leq L$ and $\lim _{j \rightarrow \infty} \eta_{j}=0$. Then, for $\left\{y\left(t ; \eta_{j}\right)\right\}_{j=0}^{\infty}$, we obtain:

$$
\begin{aligned}
y\left(t, \eta_{j}\right)= & \left.\left(y_{0}+\eta_{j}\right) e^{\frac{\rho-1}{\rho}\left(t-t_{0}\right)}+\frac{\eta_{j}}{\rho^{\alpha} \Gamma(1+\alpha)}\right) e^{\frac{\rho-1}{\rho}\left(t-t_{0}\right)}\left(t-t_{0}\right)^{\alpha} \\
& +\frac{1}{\rho^{\alpha} \Gamma(\alpha)} \int_{t_{0}}^{t} e^{\frac{\rho-1}{\rho}(t-s)}(t-s)^{\alpha-1} h\left(s, y\left(s, \eta_{j}\right)\right) d s \quad \text { for } t \in\left[t_{0}, T\right] .
\end{aligned}
$$

Now, for $t_{1}, t_{2} \in\left[t_{0}, T\right], t_{1}<t_{2}$, from (23), we have:

$$
\begin{aligned}
& \left|y\left(t_{2}, \eta_{j}\right)-y\left(t_{1}, \eta_{j}\right)\right|=\frac{1}{\rho^{\alpha} \Gamma(\alpha)} \int_{t_{0}}^{t_{2}} e^{\frac{\rho-1}{\rho}\left(t_{2}-s\right)}\left(t_{2}-s\right)^{\alpha-1} h\left(s, y\left(s, \eta_{j}\right)\right) d s \\
& -\frac{1}{\rho^{\alpha} \Gamma(\alpha)} \int_{t_{0}}^{t_{1}} e^{\frac{\rho-1}{\rho}\left(t_{1}-s\right)}\left(t_{1}-s\right)^{\alpha-1} g\left(s, u\left(s, \eta_{j}\right)\right) d s \\
& \leq \frac{1}{\rho^{\alpha} \Gamma(\alpha)} \mid \int_{t_{0}}^{t_{1}}\left(e^{\frac{\rho-1}{\rho}\left(t_{2}-s\right)}\left(t_{2}-s\right)^{\alpha-1}-e^{\frac{\rho-1}{\rho}\left(t_{1}-s\right)}\left(t_{1}-s\right)^{\alpha-1}\right) h\left(s, y\left(s, \eta_{j}\right) d s\right. \\
& +\int_{t_{1}}^{t_{2}} e^{\frac{\rho-1}{\rho}\left(t_{2}-s\right)}\left(t_{2}-s\right)^{\alpha-1} h\left(s, y\left(s, \eta_{j}\right)\right) d s \\
& \leq \frac{M}{\rho^{\alpha} \Gamma(\alpha)} \int_{t_{0}}^{t_{1}}\left|e^{\frac{\rho-1}{\rho}\left(t_{2}-s\right)}\left(t_{2}-s\right)^{\alpha-1}-e^{\frac{\rho-1}{\rho}\left(t_{1}-s\right)}\left(t_{1}-s\right)^{\alpha-1}\right| d s \\
& +\frac{M}{\rho^{\alpha} \Gamma(\alpha)} \int_{t_{1}}^{t_{2}} e^{\frac{\rho-1}{\rho}\left(t_{2}-s\right)}\left(t_{2}-s\right)^{\alpha-1} d s \\
& \leq \frac{M}{\rho^{\alpha} \Gamma(\alpha)}\left[\int_{t_{0}}^{t_{1}}\left|e^{\frac{\rho-1}{\rho}\left(t_{2}-s\right)}\left(t_{2}-s\right)^{\alpha-1}-e^{\frac{\rho-1}{\rho}\left(t_{1}-s\right)}\left(t_{1}-s\right)^{\alpha-1}\right| d s+\frac{\left(t_{2}-t_{1}\right)^{\alpha}}{\alpha}\right]
\end{aligned}
$$

and thus, $y\left(t_{2}, \eta_{j}\right)-y\left(t_{1}, \eta_{j}\right) \rightarrow 0$ as $t_{1} \rightarrow t_{2}$.

Therefore, the family of functions $\left\{u\left(t ; \eta_{j}\right)\right\}$ is equicontinuous on $\left[t_{0}, T\right]$. According to the Arzela-Ascoli theorem, there exists a subsequence $\left\{y\left(t ; \eta_{j_{k}}\right)\right\}$ and a $Y \in C\left[t_{0}, T\right]$ with $\lim _{k \rightarrow \infty} y\left(t ; \eta_{j_{k}}\right)=Y(t)$ for $t \in\left[t_{0}, T\right]$. Taking the limit in (23) as $k \rightarrow \infty$, we obtain that the function $Y(t)$ satisfies:

$$
Y(t)=y_{0} e^{\frac{\rho-1}{\rho}\left(t-t_{0}\right)}+\frac{1}{\rho^{\alpha} \Gamma(\alpha)} \int_{t_{0}}^{t} e^{\frac{\rho-1}{\rho}(t-s)}(t-s)^{\alpha-1} h(s, Y(s)) d s \quad \text { for } t \in\left[t_{0}, T\right]
$$

Therefore, $Y(t)$ is a solution of (11) for $t \in\left[t_{0}, T\right]$, and according to Condition 4 , we have $Y(t) \leq y^{*}(t)$ on $\left[t_{0}, T\right]$. From (19), we obtain $\mu(t) \leq Y(t) \leq y^{*}(t)$ on $\left[t_{0}, T\right]$.

Corollary 1. Let Condition 1 of Lemma 6 be satisfied and $V \in \Lambda\left(\left[t_{0}, T\right], \Delta\right)$ be such that the generalized proportional Caputo fractional derivative exists for any point $t \in\left[t_{0}, T\right]$, and the inequality:

$$
\left({ }_{t_{0}}^{C} D^{\alpha, \rho} V(., \tilde{x})\right)(t) \leq 0
$$

holds.

Then, $V(t, \tilde{x}(t)) \leq V\left(t_{0}, x_{0}\right) e^{\frac{\rho-1}{\rho}\left(t-t_{0}\right)} \leq V\left(t_{0}, x_{0}\right)$ for $t \in\left[t_{0}, T\right]$.

Proof. The proof follows from Remark $4\left({ }_{t_{0}}^{C} D^{\alpha, \rho} e^{\frac{\rho-1}{\rho}(.)}\right)(t)=0$, i.e., the solution of (11) with $h(t, u) \equiv 0$ and initial condition $y_{0}=V\left(t_{0}, x_{0}\right)$ is $y(t)=V\left(t_{0}, x_{0}\right) e^{\frac{\rho-1}{\rho}\left(t-t_{0}\right)}$. 
Corollary 2. Let Condition 1 of Lemma 6 be satisfied and the function $V \in \Lambda\left(\left[t_{0}, T\right], \Delta\right)$ be such that the generalized proportional Caputo fractional derivative exists for any point $t \in\left[t_{0}, T\right]$, and the inequality:

$$
\left({ }_{t_{0}}^{C} D^{\alpha, \rho} V(., \tilde{x}(.))\right)(t) \leq-\beta V(t, \tilde{x}(t))
$$

holds where $\beta>0$.

Then:

$$
V(t, \tilde{x}(t)) \leq V\left(t_{0}, x_{0}\right) e^{\frac{\rho-1}{\rho}\left(t-t_{0}\right)} E_{\alpha}\left(-\beta\left(\frac{t-t_{0}}{\rho}\right)^{\alpha}\right), \quad t \in\left[t_{0}, T\right]
$$

Proof. The proof follows from Lemma 3 about the solution of (11) with $h(t, u) \equiv-\beta u$ and initial condition $y_{0}=V\left(t_{0}, x_{0}\right)$.

Remark 5. The results of Lemma 6, Corollaries 1 and 2 are true for $T=\infty$.

\section{Main Result}

We study the stability properties of the nonlinear generalized proportional Caputo fractional differential Equation (9).

Theorem 1 (Stability). Assume:

1. The function $h \in C\left(\left[t_{0}, \infty\right) \times \mathbb{R}, \mathbb{R}\right), h(t, 0) \equiv 0$;

2. There exists a function $V \in \Lambda\left(\left[t_{0}, \infty\right), \mathbb{R}^{n}\right), V(t, 0)=0$ such that:

(i) for any initial value $x_{0} \in \mathbb{R}^{n}$, the generalized proportional Caputo fractional derivative exists and the inequality:

$$
\left({ }_{t_{0}}^{C} D^{\alpha, \rho} V(., x(.))(t) \leq h(t, V(t, x(t))), \quad t>t_{0}\right.
$$

holds where $x(t)=x\left(t ; t_{0}, x_{0}\right)$ is the solution of (9), (10);

(ii) $\quad b(\|x\|) \leq V(t, x)$ for $t \geq t_{0}, x \in \mathbb{R}^{n}$, where $b \in \mathcal{K}$;

3. The zero solution of the scalar (11) is stable.

Then, the zero solution of (9) is stable.

Proof. Let $\epsilon>0$. According to Condition 3, there exists $\delta_{1}=\delta_{1}(\epsilon)>0$ such that the inequality $\left|y_{0}\right|<\delta_{1}$ implies:

$$
\left|y\left(t ; t_{0}, y_{0}\right)\right|<b(\epsilon), \quad t \geq t_{0},
$$

where $y\left(t ; t_{0}, y_{0}\right)$ is any solution of (11). Since $V\left(t_{0}, 0\right)=0$, there exists $\delta_{2}=\delta_{2}\left(t_{0}, \delta_{1}\right)>0$ such that $V\left(t_{0}, x\right)<\delta_{1}$ for $\|x\|<\delta_{2}$. Choose $x_{0} \in \mathbb{R}^{n}:\left\|x_{0}\right\|<\delta_{2}$. Then, $V\left(t_{0}, x_{0}\right)<\delta_{1}$, and let $\tilde{x}(t)=x\left(t ; t_{0}, x_{0}\right), t \geq t_{0}$ be the corresponding solution of (9) and (10). According to Condition 2(i), the inequality $\left({ }_{t_{0}}^{C} D^{\alpha, \rho} V(., \tilde{x}()).(t) \leq h(t, V(t, \tilde{x}(t))), t \geq t_{0}\right.$ holds.

Let $y_{0}^{*}=V\left(t_{0}, x_{0}\right)$. Then, $y_{0}^{*}<\delta_{1}$, and Inequality (28) holds for the solution $y\left(t ; t_{0}, y_{0}^{*}\right)$ of (11). From Lemma 6, Remark 5, and Inequality (28), we have:

$$
V(t, \tilde{x}(t)) \leq \bar{y}(t)<b(\epsilon), \quad t \geq t_{0} ;
$$

here, $\bar{y}(t)=\bar{y}\left(t ; t_{0}, y_{0}^{*}\right)$ is the maximal solution of (11). Then, from Condition 2(ii), we obtain:

$$
b(\|\tilde{x}(t)\|) \leq V(t, \tilde{x}(t))<b(\epsilon), \quad t \geq t_{0}
$$

so the result follows.

Corollary 3. Assume $V \in \Lambda\left(\left[t_{0}, \infty\right), \mathbb{R}^{n}\right), V(t, 0)=0$, such that:

(i) For $x_{0} \in \mathbb{R}^{n}$, the generalized proportional Caputo fractional derivative exists and the inequality:

$$
\left({ }_{t_{0}}^{C} D^{\alpha, \rho} V(., x(.))(t) \leq 0, \quad t \geq t_{0}\right.
$$


holds where $x(t)=x\left(t ; t_{0}, x_{0}\right)$ is the solution of (9) and (10);

(ii) $b(\|x\|) \leq V(t, x)$ for $t \geq t_{0}, x \in \mathbb{R}^{n}$, where $b \in \mathcal{K}$.

Then, the zero solution of (9) is stable.

The stability property is also satisfied if the conditions for the Lyapunov functions are satisfied on a ball:

$$
S_{\lambda}=\left\{x \in \mathbb{R}^{n}:\|x\| \leq \lambda\right\}
$$

Theorem 2 (Stability). Assume:

1. The function $h \in C\left(\left[t_{0}, \infty\right) \times \mathbb{R}, \mathbb{R}\right), h(t, 0) \equiv 0$;

2. There exists a constants $\lambda>0$ and a function $V \in \Lambda\left(\left[t_{0}, \infty\right), S_{\lambda}\right), V(t, 0)=0$ and:

(i) For any initial value $x_{0} \in S_{\lambda}$ and $x(t)=x\left(t ; t_{0}, x_{0}\right) \in S_{\lambda}, t \geq t_{0}$, the generalized proportional Caputo fractional derivative exists and the inequality:

$$
\left({ }_{t_{0}}^{C} D^{\alpha, \rho} V(., x(.))(t) \leq h(t, V(t, x(t))), \quad t \geq t_{0}\right.
$$

holds;

(ii) $\quad b(\|x\|) \leq V(t, x) \leq a(\|x\|)$ for $t \geq t_{0}, x \in S_{\lambda}$ where $a, b \in \mathcal{K}$;

3. The zero solution of the scalar (11) is stable.

Then, the zero solution of (9) is stable.

Proof. Let $\epsilon \in(0, \lambda]$. Then, there exists $\delta_{1}=\delta_{1}(\epsilon)>0$ such that the inequality $\left|y_{0}\right|<\delta_{1}$ implies:

$$
\left|y\left(t ; t_{0}, y_{0}\right)\right|<b(\epsilon), \quad t \geq t_{0}
$$

where $y\left(t ; t_{0}, 1_{0}\right)$ is any solution of (11).

Let $\delta_{1}=\min \{\varepsilon, b(\varepsilon)\}$.

Since $a \in \mathcal{K}$, there exists $\delta_{2}=\delta_{2}\left(\delta_{1}\right)>0$ such that if $\xi<\delta_{2}$, then $a(\xi)<\delta_{1}$. Let $\delta=\min \left\{\varepsilon, \delta_{2}\right\}$.

Choose $x_{0}$ : $\left\|x_{0}\right\|<\delta$, and let $\tilde{x}(t)=x\left(t ; t_{0}, x_{0}\right), t \geq t_{0}$ be the corresponding solution of (9) and (10).

We prove:

$$
\|\tilde{x}(t)\|<\varepsilon, \quad t \geq t_{0} .
$$

Assume this is false. Then, there exists a point $\tau>t_{0}$ such that $\|\tilde{x}(t)\|<\varepsilon, t \in\left[t_{0}, \tau\right)$, and $\|\tilde{x}(\tau)\|=\varepsilon$, i.e., $\tilde{x}(t) \in S_{\lambda}, t \in\left[t_{0}, \tau\right]$.

Let $y_{0}^{*}=V\left(t_{0}, x_{0}\right)$. Then $y_{0}^{*}<a\left(\left\|x_{0}\right\|\right), a\left(\delta_{2}\right)<\delta_{1}$, and Inequality (31) holds for the solution $y\left(t ; t_{0}, y_{0}^{*}\right)$ of the scalar FrDE (11). Then, from Lemma 6, we have:

$$
V(t, \tilde{x}(t)) \leq \bar{y}(t)<b(\epsilon), \quad t \in\left[t_{0}, \tau\right]
$$

here, $\bar{y}(t)=\bar{y}\left(t ; t_{0}, y_{0}^{*}\right)$ is the maximal solution of (11). Then, from Condition 2(ii), we obtain:

$$
b(\varepsilon)=b(\|\tilde{x}(\tau)\|) \leq V(\tau, \tilde{x}(\tau))<b(\epsilon) .
$$

The obtained contradiction proves Inequality (32).

Theorem 3 (Asymptotic stability). Let $V \in \Lambda\left(\mathbb{R}_{+}, \mathbb{R}^{n}\right)$ and:

(i) For any initial value $x_{0} \in S_{\lambda}$ and any solution $x(t)=x\left(t ; t_{0}, x_{0}\right) \in S_{\lambda}, t \geq t_{0}$ of (9), (10), the generalized proportional Caputo fractional derivative exists and the inequality:

$$
\left({ }_{t_{0}}^{C} D^{\alpha, \rho} V(., x(.))(t) \leq-c V(t, x(t)), \quad t \geq t_{0}\right.
$$

holds where $c>0$;

(ii) $b(\|x\|) \leq V(t, x) \leq a(\|x\|)$ for $t \geq t_{0}, x \in S_{\lambda}$ where $a, b \in \mathcal{K}$.

Then, the zero solution of (9) is asymptotically stable. 
Proof. According to Theorem 2, the zero solution of (9) is stable. Thus, there exists a constant $A=A(\lambda) \in(0, \lambda)$ such that the inequality $\left\|\tilde{x}_{0}\right\|<A$ implies:

$$
\left\|x\left(t ; t_{0}, \tilde{x}_{0}\right)\right\|<\lambda \text { for } t \geq t_{0}
$$

where $x\left(t ; t_{0}, \tilde{x}_{0}\right)$ is a solution of (9) and (10).

Let $\beta \in(0, A]: a(\beta) \leq b(A)$, and choose $x_{0} \in \mathbb{R}^{n}:\left\|x_{0}\right\|<\beta$. Consider the solution $\tilde{x}(t)=x\left(t ; t_{0}, x_{0}\right)$ of (9) with initial condition $x\left(t_{0}\right)=x_{0}$. Then, $b\left(\left\|x_{0}\right\|\right) \leq a\left(\left\|x_{0}\right\|\right)<$ $a(B)<b(A)$, i.e., $\left\|x_{0}\right\|<A$, and therefore, $\tilde{x}(t) \in S_{\lambda}, t \geq t_{0}$.

According to Corollary 2, we obtain:

$$
V(t, \tilde{x}(t)) \leq V\left(t_{0}, x_{0}\right) e^{\frac{\rho-1}{\rho}\left(t-t_{0}\right)} E_{\alpha}\left(-c\left(\frac{t-t_{0}}{\rho}\right)^{\alpha}\right), \quad t \geq t_{0} .
$$

Choose an arbitrary number $\varepsilon \in(0, B)$. Consider the function $\eta(t)=e^{\frac{\rho-1}{\rho} t} E_{\alpha}\left(-c\left(\frac{t}{\rho}\right)^{\alpha}\right)$, which is decreasing. Thus, there exists a number $T>0$ such that $\eta(t)<\frac{b(\varepsilon)}{a(B)}$ for $t>T$. Thus,

$$
\begin{aligned}
b(\|\tilde{x}(t)\|) & \leq V(t, \tilde{x}(t)) \leq V\left(t_{0}, x_{0}\right) e^{\frac{\rho-1}{\rho}\left(t-t_{0}\right)} E_{\alpha}\left(-c\left(\frac{t-t_{0}}{\rho}\right)^{\alpha}\right) \\
& \leq a\left(\left\|x_{0}\right\|\right) e^{\frac{\rho-1}{\rho}\left(t-t_{0}\right)} E_{\alpha}\left(-c\left(\frac{t-t_{0}}{\rho}\right)^{\alpha}\right)<b(\epsilon), \quad t \geq T .
\end{aligned}
$$

Therefore, $\|\tilde{x}(t)\|<\varepsilon, \quad t \geq t_{0}+T$ holds, and the zero solution of (9), (10) is attractive.

\section{Applications}

Example 2. Consider the following system of fractional differential equations with the GPFD:

$$
\begin{aligned}
& \left({ }_{0}^{C} D^{\alpha, \rho} x_{1}\right)(t)=-(1-\rho) \frac{\left(1+t^{2}\right)}{\left(t^{2}+2\right)} x_{1}(t)-g_{2}(t) x_{2}(t), \\
& \left({ }_{0}^{C} D^{\alpha, \rho} x_{2}\right)(t)=-(1-\rho) \frac{\left(1+t^{2}\right)}{\left(t^{2}+2\right)} x_{2}(t)+g_{2}(t) x_{1}(t) \quad \text { for } t \geq 0
\end{aligned}
$$

with initial condition:

$$
x_{1}(0)=x_{1}^{0} \text { and } x_{2}(0)=x_{2}^{0}
$$

where $x_{1}, x_{2} \in \mathbb{R}, \alpha \in(0,1), \rho \in(0,1]$, and $g_{2} \in C\left(\mathbb{R}_{+}, \mathbb{R}\right)$ is an arbitrary function.

Note that $(1-\rho) \frac{\left(1+t^{2}\right)}{\left(t^{2}+2\right)} \in[0.5(1-\rho),(1-\rho))$ for $t \geq 0$.

Consider $V\left(t, x_{1}, x_{2}\right)=x_{1}^{2}+x_{2}^{2}$ for $t \in \mathbb{R}_{+}, x=\left(x_{1}, x_{2}\right) \in \mathbb{R}^{2}$.

For any $x_{1}, x_{2} \in \mathbb{R}: x=\left(x_{1}, x_{2}\right)$, apply Lemma 4 , and obtain:

$$
\begin{aligned}
& \left({ }_{t_{0}}^{C} D^{\alpha, \rho} V\left(., x_{1}(.), x_{2}(.)\right)\right)(t)=\left({ }_{t_{0}}^{C} D^{\alpha, \rho} x_{1}^{2}(.)\right)(t)+\left({ }_{t_{0}}^{C} D^{\alpha, \rho} x_{2}^{2}(.)\right)(t) \\
& \leq 2 x_{1}(t)\left({ }_{t_{0}}^{C} D^{\alpha, \rho} x_{1}(.)\right)(t)+2 x_{2}(t)\left({ }_{t_{0}}^{C} D^{\alpha, \rho} x_{2}(.)\right)(t) \\
& =-2(1-\rho) \frac{\left(1+t^{2}\right)}{\left(t^{2}+2\right)} V\left(t, x_{1}, x_{2}\right) \leq-(1-\rho) V\left(t, x_{1}, x_{2}\right) .
\end{aligned}
$$

Case 1. Let $\rho=1$ (Caputo fractional differential equation). From (36), we obtain the inequality $\left.{ }_{t_{0}}^{C} D^{\alpha, \rho} V\left(., x_{1}(),. x_{2}().\right)\right)(t) \leq 0$, and according to Corollary 3 , the zero solution of $(35)$ is stable.

Case 2. Let $\rho \in(0,1)$, and from (36), according to Theorem 3, the zero solution of (35) is asymptotically stable.

Therefore, the stability properties depend on the parameter $\rho$ of the generalized proportional fractional derivative. 
Consider the corresponding to (35) system with ordinary derivatives:

$$
\begin{aligned}
& x_{1}^{\prime}(t)=-(1-\rho) \frac{\left(1+t^{2}\right)}{\left(t^{2}+2\right)}(t) x_{1}-g_{2}(t) x_{2}, \\
& x_{2}^{\prime}(t)=-(1-\rho) \frac{\left(1+t^{2}\right)}{\left(t^{2}+2\right)}(t) x_{2}+g_{2}(t) x_{1}, \text { for } t \geq 0, \rho \in(0,1), \\
& x_{1}(0)=x_{1}^{0} \text { and } x_{2}(0)=x_{2}^{0} .
\end{aligned}
$$

Case 3. Let $\rho=1$. Then, the solutions are not stable (see Figure 1) (compare with Case 1).

Case 4. Let $\rho=0.9$. The zero solution is asymptotically stable (see Figure 2 with $x_{1}(0)=$ $x_{2}(0)=0.52$ and $\left.g_{2}(t) \equiv-1\right)$.

Therefore, the type of the derivative (ordinary derivative, or Caputo fractional derivative, or the GPFD) in the differential equation has a significant influence on the behavior of the solutions.

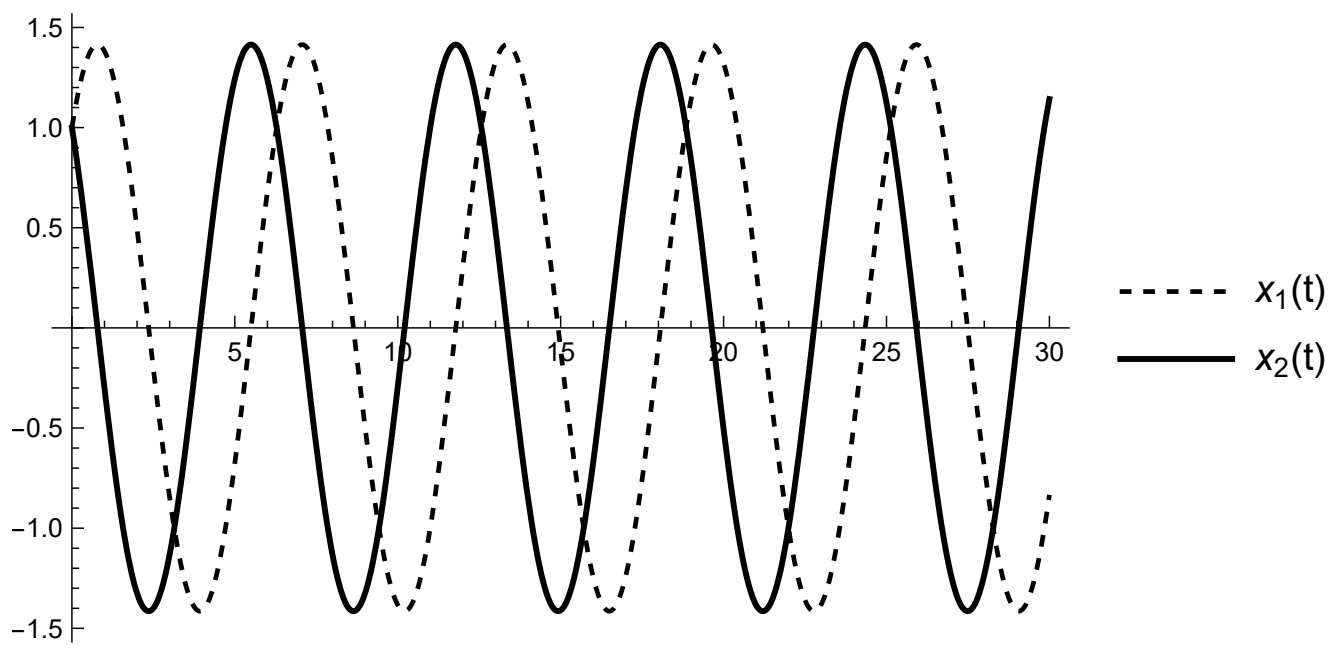

Figure 1. Graph of the solutions (37) for $\rho=1, g_{2}(t) \equiv-1$.

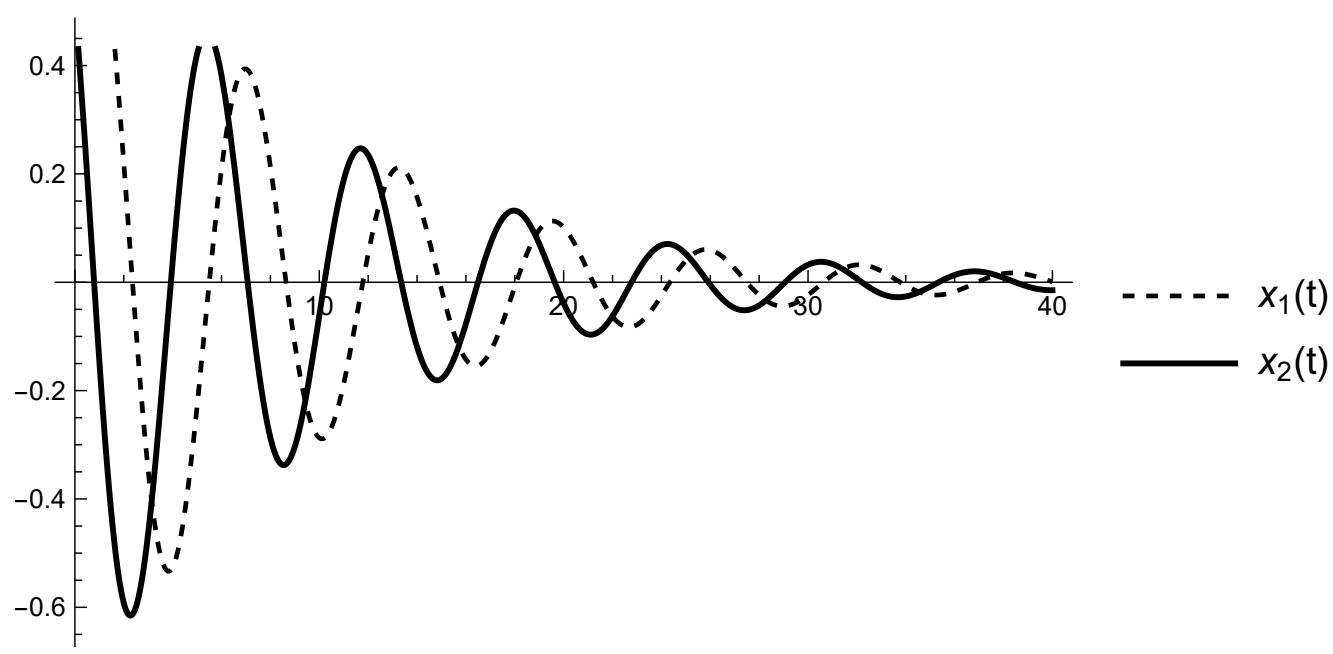

Figure 2. Graph of the solutions (37) for $\rho=0.9, g_{2}(t) \equiv-1$.

Example 3. Consider the following system of nonlinear fractional differential equations with the GPFD:

$$
\begin{aligned}
& \left({ }_{0}^{C} D^{\alpha, \rho} x_{1}\right)(t)=a \sin \left(x_{1}(t)\right)-g(t) x-2(t), \\
& \left({ }_{0}^{C} D^{\alpha, \rho} x_{2}\right)(t)=g(t) x_{1}(t)+a \sin \left(x_{2}(t)\right) \quad \text { for } t \geq 0, \alpha \in(0.1), \rho \in(0,1),
\end{aligned}
$$


with initial condition,

$$
x_{1}(0)=x_{1}^{0} \text { and } x_{2}(0)=x_{2}^{0}
$$

where $x_{1}, x_{2} \in \mathbb{R}, g \in C([0, \infty), \mathbb{R})$ is an arbitrary function and $a>0: a<0.5(1-\rho)^{\alpha}$. Consider $V\left(t, x_{1}, x_{2}\right)=x_{1}^{2}+x_{2}^{2}$ for $t \in \mathbb{R}_{+}, x_{1}, x_{2} \in \mathbb{R}$.

For any $x_{1}, x_{2} \in \mathbb{R}$, apply Lemma 4 , and obtain:

$$
\begin{aligned}
& \left({ }_{t_{0}}^{C} D^{\alpha, \rho} V\left(., x_{1}(.), x_{2}(.)\right)\right)(t)=\left({ }_{t_{0}}^{C} D^{\alpha, \rho} x_{1}^{2}(.)\right)(t)+\left({ }_{t_{0}}^{C} D^{\alpha, \rho} x_{2}^{2}(.)\right)(t) \\
& \leq 2 x_{1}(t)\left({ }_{t_{0}}^{C} D^{\alpha, \rho} x_{1}(.)\right)(t)+2 x_{2}(t)\left({ }_{t_{0}}^{C} D^{\alpha, \rho} x_{2}(.)\right)(t) \\
& =2 a x_{1}(t) \sin \left(x_{1}(t)\right)-2 g(t) x_{1}(t) x_{2}(t)+2 x_{2}(t) g(t) x_{1}(t)+2 a x_{2}(t) \sin \left(x_{2}(t)\right) \\
& \leq 2 a\left(x_{1}^{2}(t)+x_{2}^{2}(t)\right)=2 a V\left(t, x_{1}, x_{2}\right) .
\end{aligned}
$$

Consider the scalar fractional Equation (11) with $h(t, y)=2 a y, y \in \mathbb{R}$, the solution of which according to Lemma 3 with $\lambda=2 a$ is given by $y(t)=y_{0} e^{\frac{\rho-1}{\rho} t} E_{\alpha}\left(2 a\left(\frac{t}{\rho}\right)^{\alpha}\right)$. According to Theorem 1.2 [23], for every $\alpha \in(0,1)$, the function, $\frac{e^{t}}{\alpha}-E_{\alpha}\left(t^{\alpha}\right)$ is completely monotonic and $E_{\alpha}\left(t^{\alpha}\right)<\frac{e^{t}}{\alpha}, t \geq 0$. Therefore, $E_{\alpha}\left(2 a\left(\frac{t}{\rho}\right)^{\alpha}\right)=E_{\alpha}\left(\left(\frac{\sqrt[\alpha]{2 a} t}{\rho}\right)^{\alpha}\right) \leq \frac{e^{\frac{\sqrt[\alpha]{2 a} t}{\rho}}}{\alpha}$ and $|y(t)| \leq\left|y_{0}\right| e^{\frac{\rho-1}{\rho} t} \frac{e^{\frac{\sqrt[\alpha]{2 a} t}{\rho}}}{\alpha}=\frac{\left|y_{0}\right|}{\alpha} e^{\frac{\rho-1+\sqrt[\alpha]{2 a}}{\rho} t}$.

Thus, the zero solution of the considered particular case of the scalar (11) is stable since $\rho-1+\sqrt[\alpha]{2 a}<0$. According to Theorem 1, the zero solution of the nonlinear system (38) is stable.

\section{Conclusions}

A system of nonlinear equations with the GPFD was considered. The stability properties of the zero solution were studied using Lyapunov functions and their GPFDs. First, some comparison results with scalar nonlinear fractional differential equations with the GPFD and Lyapunov functions were established and several sufficient conditions for stability and asymptotic stability were obtained and illustrated with examples. In the future, we hope to study other types of stability for nonlinear fractional differential equations with the GPFD and apply them to some new models.

Author Contributions: Conceptualization, R.A., S.H. and D.O.; methodology, R.A., S.H. and D.O.; validation, R.A., S.H. and D.O.; formal analysis, R.A., S.H. and D.O.; writing-original draft preparation, R.A., S.H. and D.O.; writing - review and editing, R.A., S.H. and D.O.; funding acquisition, S.H. All authors have read and agreed to the published version of the manuscript.

Funding: This work is partially supported by the Bulgarian National Science Fund under Project KP-06-N32/7.

Institutional Review Board Statement: Not applicable.

Informed Consent Statement: Not applicable.

Data Availability Statement: Not applicable.

Conflicts of Interest: The authors declare no conflict of interest.

\section{References}

1. Trigeassou, J.C.; Maamri, N.; Sabatier, J.; Oustaloup, A. A Lyapunov approach to the stability of fractional differential equations. Signal Process. 2011, 91, 437-445. [CrossRef]

2. Aguila-Camacho, N.; Duarte-Mermoud, M.A.; Gallegos, J.A. Lyapunov functions for fractional order systems. Comm. Nonlinear Sci. Numer. Simul. 2014, 19, 2951-2957. [CrossRef]

3. Burton, T.A. Fractional Differential equations and Lyapunov functionals. Nonlinear Anal. Theory Methods Appl. 2011, 74, 5648-5662. [CrossRef]

4. Duarte-Mermoud, M.A.; Aguila-Camacho, N.; Gallegos, J.A.; Castro-Linares, R. Using general quadratic Lyapunov functions to prove Lyapunov uniform stability for fractional order systems. Commun. Nonlinear Sci. Numer. Simul. 2015, 22, 650-659. [CrossRef] 
5. Hu, J.B.; Lu, G.P.; Zhang, S.B.; Zhao, L.-D. Lyapunov stability theorem about fractional system without and with delay. Commun. Nonlinear Sci. Numer. Simulat. 2015, 20, 905-913. [CrossRef]

6. Li, Y.; Chen, Y.; Podlubny, I. Stability of fractional-order nonlinear dynamic systems: Lyapunov direct method and generalized Mittag-Leffler stability. Comput. Math. Appl. 2010, 59, 1810-1821. [CrossRef]

7. Li, C.P.; Zhang, F.R. A survey on the stability of fractional differential equations. Eur. Phys. J. Spec. Top. 2011, 193, 27-47. [CrossRef]

8. Jarad, F.; Abdeljawad, T.; Alzabut, J. Generalized fractional derivatives generated by a class of local proportional derivatives. Eur. Phys. J. Spec. Top. 2017, 226, 3457-3471. [CrossRef]

9. Abbas, M.I.; Ragusa, M.A. On the hybrid fractional differential equations with fractional proportional derivatives of a function with respect to a certain function. Symmetry 2021, 13, 264. [CrossRef]

10. Alzabut, J.; Abdeljawad, T.; Jarad, F.; Sudsutad, W. A Gronwall inequality via the generalized proportional fractional derivative with applications. J. Ineq. Appl. 2019, 2019, 101. [CrossRef]

11. Hristova, S.; Abbas, M.I. Explicit solutions of initial-value problems for fractional generalized proportional differential equations with and without impulses. Symmetry 2021, 13, 996. [CrossRef]

12. Jarad, F.; Abdeljawad, T. Generalized fractional derivatives and Laplace transform. Discret. Contin. Dyn. Syst.-S 2020, 13, 709-722. [CrossRef]

13. Laadjal, Z.; Abdeljawad, T.; Jarad, F. On existence-uniqueness results for proportional fractional differential equations and incomplete gamma functions. Adv. Differ. Equ. 2020, 2020, 641. [CrossRef]

14. Almeida, R.; Agarwal, R.P.; Hristova, S.; O’Regan, D. Quadratic Lyapunov functions for stability of generalized proportional fractional differential equations with applications to neural networks. Axioms 2021, 10, 322. [CrossRef]

15. Li, C.; Qian, D.; Chen, Y. On Riemann-Liouville and Caputo Derivatives. Discret. Dyn. Nat. Soc. 2011, 2011, 562494. [CrossRef]

16. Baleanu, D.; Mustafa, O.G. On the global existence of solutions to a class of fractional differential equations. Comput. Math. Appl. 2010, 59, 1835-1841. [CrossRef]

17. Das, S. Functional Fractional Calculus; Springer: Berlin/Heidelberg, Germany, 2011.

18. Diethelm, K. The Analysis of Fractional Differential Equations; Springer: Berlin/Heidelberg, Germany, 2010.

19. Podlubny, I. Fractional Differential Equations; Academic Press: San Diego, CA, USA, 1999.

20. Samko, G.; Kilbas, A.A.; Marichev, O.I. Fractional Integrals and Derivatives: Theory and Applications; Gordon and Breach: Philadelphia, PA, USA, 1993.

21. Abbas, M.I.; Hristova, S. Existence results of nonlinear generalized proportional fractional differential inclusions via the diagonalization technique. AIMS Math. 2021, 6, 12832-12844. [CrossRef]

22. Abbas, M.I.; Hristova, S. On the Initial Value Problems for Caputo-Type Generalized Proportional Vector-Order Fractional Differential Equations. Mathematics 2021, 9, 2720. [CrossRef]

23. Simon, T. Mittag-Leffler functions and complete monotonicity. Integral Transf. Spec. 2015, 26, 36-50. [CrossRef] 